This journal is the official publication of Bangladesh Society of Physiologists (BSP)

Web URL: www.banglajol.info/index.php/JBSP

Abstracted /indexed in Index Copernicus, Director of Open Access Journal, HINARI Index Medicus for South East Asia Region, Google Scholar, 12OR, infobse index, Open J gate, Cite factor, Scientific indexing services

pISSN-1983-1213; e-ISSN-2219-7508

\title{
Article
}

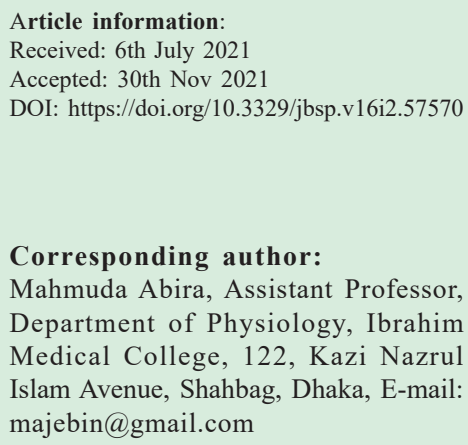

This article is open access licensed under CC BY NC SA which allows readers copy, distribute, display, and perform the work and make derivative works based on it only for noncommercial purposes.

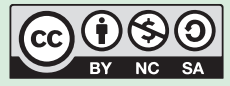

\section{Red blood cell distribution width: a promising index for estimating disease activity of Systemic lupus erythematosus}

\author{
Mahmuda Abira' ${ }^{1}$ Qazi Shamima Akhter ${ }^{2}$, Mohammad \\ Sirajul Islam ${ }^{3}$ \\ 1. Department of Physiology, Ibrahim Medical College, Dhaka \\ 2. Department of Physiology, Dhaka Medical College, Dhaka \\ 3. Department of Medicine, Sir Salimullah Medical College, Dhaka
}

\section{Abstract}

Background: Systemic lupus erythematosus (SLE) is a chronic inflammatory disease and is associated with considerable morbidity. Elevation in red cell distribution width (RDW), a simple routinely done investigation, could be a prognostic marker in SLE patients. Objective: To assess red blood cell distribution width for estimating disease activity in patients with SLE. Methods: This cross-sectional study was conducted in the Department of Physiology, Dhaka Medical College, Dhaka from July 2016 to June 2017. For this study, 30 SLE patients, aged 18 to 55 years, were considered as the study group and 30 aged matched healthy subjects were considered as control group. SLE Disease Activity Index (SLEDAI) score was used to assess disease activity. RDW and ESR were also estimated. Independent samples $t$-test, Chi Square test, Pearson's correlation co-efficient (r) test, Regression analysis, Diagnostic accuracy test and ROC curve analysis were performed as applicable.. Results: In this study, RDW and ESR was significantly ( $p=0.000)$ higher in SLE patients in comparison to that of control. A significant association was observed among RDW, ESR and active disease (OR; 5.33, 95\% CI; 0.78 to 36.33 and OR; 5.66, 95\% CI; 0.75 to $42.36, p=0.001)$. RDW was positively correlated with SLEDAI score and ESR in patients with SLE. It was $84.2 \%$ sensitive and $72.7 \%$ specific for diagnosing active disease. Conclusion: This study concludes that RDW is increased in SLE patients and can be served as a useful index to estimate the disease activity.

Key words: Systemic lupus erythematosus (SLE), Red cell distribution width (RDW), Erythrocyte sedimentation rate (ESR), Systemic Lupus Erythematosus Disease Activity Index (SLEDAI) 
Introduction

$\mathbf{S}$ ystemic lupus erythematosus (SLE) is a chronic systemic autoimmune rheumatic disease of variable severity with a period of remission and flares that can be fatal if not treated early. The disease starts with a preclinical phase characterized by production of autoantibodies. These antibodies form immune complexes which may contribute to formation of all clinical and laboratory manifestations. Early cell and organ damage is primarily mediated by tissue-binding auto-antibodies and late damage are usually related to complications of long standing disease and immunosuppressive therapy. ${ }^{1-2}$

The cause of SLE is incompletely understood but genetic, hormonal, abnormal immunologic background and the environmental factors especially ultraviolet rays play an important role. . $^{3-}$ ${ }^{4}$ A greater incidence of SLE in women has always been reported (49.6 per 100,000) and it frequently occurs during the child bearing age being 1 in 500. Male were found to have a biphasic pattern with prevalence of 6 per 100,000 between the age of 30 to 39 years and 60 to 69 years. ${ }^{5-6}$

Causes of death have been different among SLE patients in different countries. Active disease, infection or cardiovascular involvement is the cause of death in US, Denmark and other European countries ${ }^{7-9}$. While infections and active SLE were leading causes of death in Asia. ${ }^{10}$ The precise evaluation of disease activity is of great importance to assess disease progression and prognosis for SLE patients. Many diverse inflammation indices have been used to evaluate inflammatory status in SLE and as a marker of disease activity, such as erythrocyte sedimentation rate (ESR) C-reactive protein (CRP) and Anti-double stranded DNA antibody (antidsDNA). ${ }^{11}$ The red blood cell distribution width (RDW) is widely available, inexpensive and easily repeatable, novel, accurate inflammatory biomarker which are easily measured and sensitive to changes in disease activity. ${ }^{12}$
RDW is a morphological guide of circulating red blood cells (RBC) and represent a wide-ranging heterogeneity of size and volume of RBC in peripheral blood (known as anisocytosis). It can be easily estimated by the modern hematological analyzers. RDW gives the coefficient of variation of the RBC volume in percentage and thereby expresses the width of the volume curve.${ }^{13}$ It varies with diseases that alter the erythropoiesis and the composition of RBC. Any condition that increases the release of immature $\mathrm{RBC}$ from the bone marrow will cause an increase in RDW. Relatively larger volume of immature cells contributes to a greater span of the volume distribution curve. ${ }^{14}$

Conventionally, RDW is used in the differential diagnosis of anemia. Because it becomes raised earlier than other blood parameters. It is also helpful for initial diagnosis of nutritional deficiency. ${ }^{15}$ Megaloblastic anemia also presents with high values of both MCV and RDW and iron deficiency anemia is characterized by a high RDW and low MCV. ${ }^{13}$ Recent study demonstrated association of increased RDW with nonhematological diseases such as thyroid disfunction, inflammatory bowel disease, Behcet's disease, rheumatoid arthritis and SLE. ${ }^{16}$ Very few studies investigated about the importance of RDW to assess the disease activity of SLE . Therefore, the present study has been designed to explore the feasibility of RDW for estimating disease activity and prognosis in patients with SLE.

\section{Methods}

This cross-sectional study was conducted in the Department of Physiology, Dhaka Medical College, Dhaka from July 2016 to June 2017. For this study, 30 diagnosed SLE patients aged 18 to 55 years, duration of disease $\leq 5$ years were recruited to this study by purposive sampling. They were diagnosed by rheumatologist of Dhaka Medical College Hospital (DMCH) based on American College of Rheumatology (ACR)

Volume 16 No. 2 December 2021: 95-103 
Criteria. ${ }^{4}$ All the patients were selected from SLE clinic of DMCH. 30 age, sex and BMI matched healthy subjects without having SLE were considered as control group for comparison. They were selected by personal contact from different areas of Dhaka city. ACR criteria includes 16 points based on clinical and laboratory judgment. The clinical features are malar rash, discoid rash, photosensitivity, alopecia, oral/ nasal ulcers, polyarthralgia or myalgia, polyarthritis, pleurisy or pericarditis, peritonitis, hematuria, proteinuria etc. and the hematological features are leukopenia, thrombocytopenia, hemolytic anemia etc. The patients with 4 points out of 16 have definite diagnosis of SLE, with 3 points were highly suggestive for SLE, with 2 points was probable SLE and with one-point was possible SLE. ${ }^{4}$ SLEDAI score ${ }^{17}$ was used to assess disease activity. SLEDAI score of each patient was evaluated and allocated a clinical activity score for each assessment according to the following scale: $0=$ no activity; $1=$ mild activity with no therapeutic intervention; 2 = activity, but improvement from previous visit; 3 $=$ persistent activity $/$ refractory to treatment; $4=$ flare. ${ }^{15}$ According to the SLEDAI score, we categorized the SLE patients into 2 groups such as patients with mild/inactive disease if score was $\leq 9$ and severe/active disease if score was $>9$. The patients having history of liver disease, renal disease rheumatoid arthritis, ankylosing spondylitis, inflammatory bowel disease, psoriasis and malignant disease, history of taking anticoagulant, chemotherapy and recent history of blood transfusion were excluded from the study. After selection, the nature, purpose and benefit of the study were explained to each subject in details. An Informed written consent was taken from the participants. The research work was carried out after obtaining ethical clearance from Ethical Review Committee of Dhaka Medical College, Dhaka. Before taking blood, detailed family and medical history were taken. Anthropometric measurement of the subjects was done and blood pressure was measured. All the information was recorded in a prefixed questionnaire. Blood samples were collected under asepsis for estimation of RDW and ESR. RDW was estimated by Automated Hematology Analyzer (Sysmex XT-2000) ${ }^{18}$ and ESR was estimated by Westergren's method. ${ }^{19}$

Data were expressed as frequency, percentage, mean $\pm \mathrm{SE}$ and Independent samples $t$-test, Chi Square test and Pearson's correlation co-efficient ( $\mathrm{r}$ ) test were performed as applicable. Univariate regression analysis was done to observed Odds Ratio (OR) and the association of RDW and ESR in SLE patients and healthy adult subjects. Multivariate logistic regression analysis was performed by adjusting age to observe OR and the association of RDW and ESR with SLEDAI score in SLE patients. Diagnostic accuracy of RDW and ESR was analyzed and the area under the ROC curve (receiver operating characteristic curve) also accessed. 95\% confident interval (CI) was calculated and $p$ value $<0.05$ was accepted as level of significance. Statistical analyses were performed by using IBM SPSS (statistical package for social sciences) Statistics for Windows version 26.0.

\section{Results}

In this study, the control and the study groups were age, sex, BMI and BP matched (Table I).

In this study, the mean $\pm \mathrm{SE}$ of RDW and ESR was significantly $(p=0.000)$ higher in SLE patients in comparison to control. SLE patients were more likely to have RDW and ESR alteration than control (Table II). in Majority SLE patients had SLEDAI score $>9$ (active disease) (Figure 1). RDW and ESR was significantly $(p<0.05)$ higher in active SLE than inactive SLE (Figure 2,3) and showed a significant association among RDW, ESR and active disease (OR; $5.33,95 \% \mathrm{CI} ; 0.78$ to 36.33 and OR; $5.66,95 \% \mathrm{CI} ; 0.75$ to $42.36, p=0.001$ ). 
Table I: General characteristics of the subjects in both groups $(\mathrm{N}=60)$

\begin{tabular}{lccc}
\hline Parameters & SLE $(\mathrm{n}=30)$ & Control $(\mathrm{n}=30)$ & $p$ value \\
\hline Age (years) & & & \\
$\leq 40$ no.(\%) & $19(63.33 \%)$ & $18(60 \%)$ & 0.791 \\
$>40-55$ no.(\%) & $11(36.67 \%)$ & $12(40 \%)$ & \\
& $35.10 \pm 1.93$ & $34.89 \pm 2.51$ & 0.718 \\
Sex no.(\%) & & \\
$\quad$ & & & \\
$\quad$ Male & $2(6.7 \%)$ & $26(13.3 \%)$ & 0.389 \\
$\quad$ Female & $28(93.3 \%)$ & $21.91 \pm 1.18$ & 0.387 \\
BMI $\left(\mathrm{kg} / \mathrm{m}^{2}\right)$ & $20.34 \pm 1.08$ & $122.30 \pm 2.37$ & 0.347 \\
Systolic BP $(\mathrm{mmHg})$ & $123.60 \pm 1.32$ & $79.67 \pm 1.76$ & 0.197 \\
Diastolic BP $(\mathrm{mmHg})$ & $81.67 \pm 1.45$ &
\end{tabular}

Data were shown as mean \pm SE. Statistical analysis was done by Independent samples $t$-test and Chi Square test (frequency, \%). SLE-Systemic lupus erythematosus, Control-Healthy subjects, BMI- Body mass index, BPblood pressure, $\mathrm{N}=$ total number of subjects, $\mathrm{n}=$ number of subjects in each group

Table II: RDW, ESR and SLEDAI score of the subjects in both groups $(\mathrm{N}=60)$

\begin{tabular}{lccccc}
\hline Parameters & SLE $(\mathrm{n}=30)$ & Control $(\mathrm{n}=30)$ & Mean difference $(95 \% \mathrm{CI})$ & OR $(95 \% \mathrm{CI})$ & $p$ value \\
\hline RDW (\%) & $17.99 \pm 0.85$ & $12.93 \pm 0.21$ & $5.06(3.30$ to 6.80$)$ & $3.73(2.25$ to 6.18$)$ & $0.000^{* * *}$ \\
ESR (mm & $47.60 \pm 2.91$ & $9.47 \pm 0.73$ & $38.13(32.13$ to 44.14$)$ & $7.00(3.11$ to 15.76$)$ & $0.000^{* * *}$ \\
in $1^{\text {st }}$ hour $)$ & & & & & \\
\hline
\end{tabular}

Data were shown as mean \pm SE. Statistical analysis was done by Independent samples $t$-test and Univariate regression analysis. SLE-Systemic lupus erythematosus, Control- Healthy subjects, RDW- Red Cell Distribution Width, ESR- Erythrocyte sedimentation rate, SLEDAI- Systemic lupus erythematosus disease activity index, $* * * \mathrm{p}<0.001, \mathrm{~N}=$ total number of subjects, $\mathrm{n}=$ number of subjects in each group

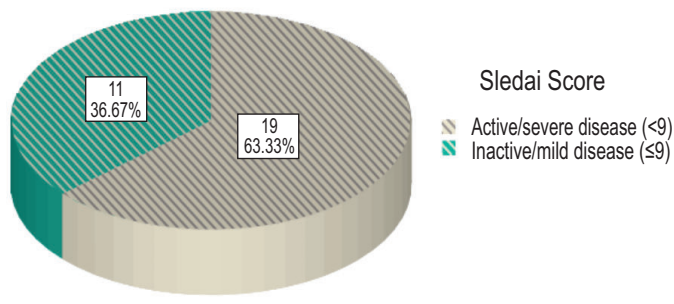

Figure 1: Distribution of study subjects according to SLEDAI score presenting that majority $(19 ; 63.33 \%)$ of the SLE patients had SLEDAI score $>9$. SLEDAI $=$ Systemic lupus erythomatosus disease activity index.

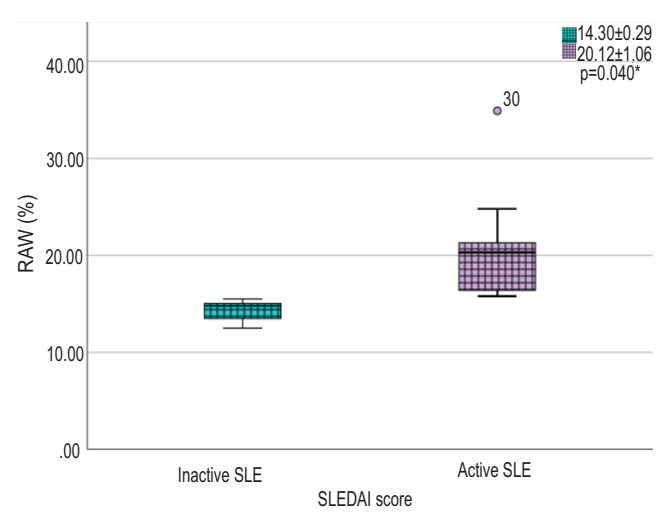

Figure 2: RDW of SLE patients presenting that RDW was increased more in active SLE than inactive SLE. RDW-Red Cell Distribution Width, ${ }^{*} \mathrm{p}<0.05$

Volume 16 No. 2 December 2021: 95-103 


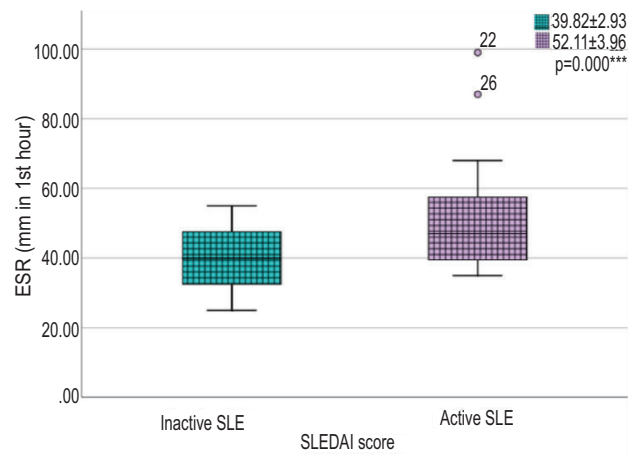

Figure 3: ESR of SLE patients presenting that ESR was increased more in active SLE than inactive SLE. ESR $=$ Erythrocyte sedimentation rate, $* * *=\mathrm{P}<0.001$

Correlation analysis showed, significant $(p<0.05)$ positive correlation among RDW, ESR and SLEDAI score (Figure 4, 5 \& 6).

RDW was $84.2 \%$ sensitive and $72.7 \%$ specific for diagnosing active disease. Positive predictive value (PPV) was $84.2 \%$ and negative predictive value (NPV) was $72.7 \%$. ESR was $78.9 \%$ sensitive and $81.8 \%$ specific for diagnosing active disease. PPV was $88.2 \%$ and NPV was $69.2 \%$ (Table III).

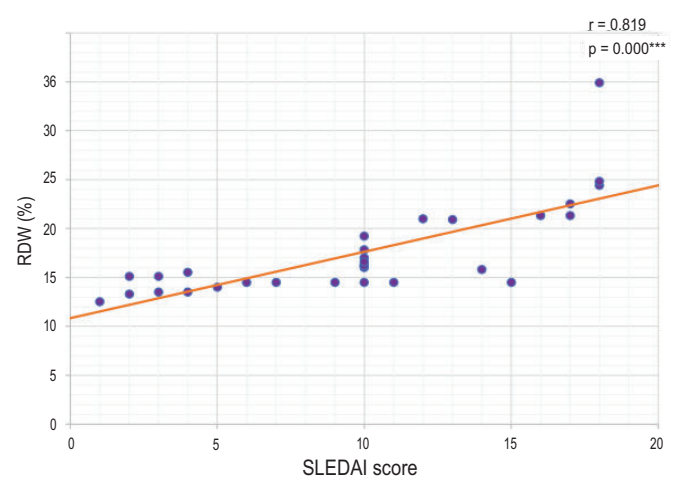

Figure 4: Correlation of RDW with SLEDAI score in SLE patients presenting that there is positive correlation between RDW and SLEDAI score. RDW $=$ Red Cell Distribution Width, SLEDAI = Systemic lupus erythematosus disease activity index, ${ }^{* * *} \mathrm{p}<0.001$

Volume 16 No. 2 December 2021:95-103
Diagnostic ability is measured by the area under the ROC curve. The area of RDW and ESR under the ROC curve is $0.837(95 \% \mathrm{CI}: 0.666$ to $1.000 ; \mathrm{p}<0.01)$ and 0.883 (95\% CI: 0.734 to 1.000 ; $\mathrm{p}<0.01)$ and considered as excellent for separating active SLE from inactive SLE patients (Figure 7).

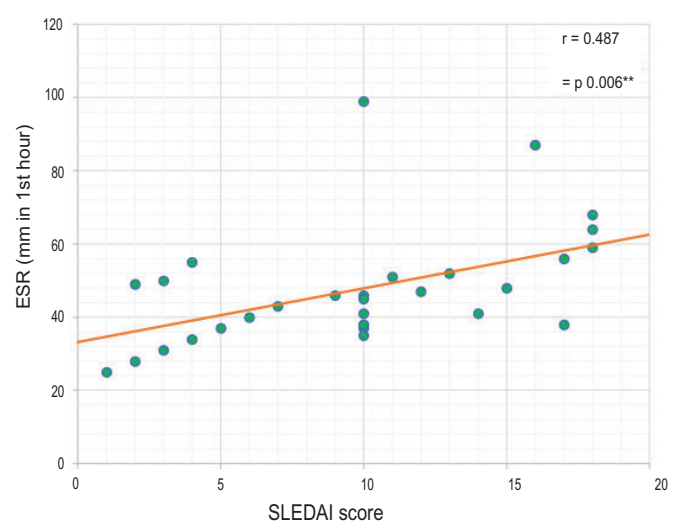

Figure 5: Correlation of ESR with SLEDAI score in SLE patients presenting that there is positive correlation between ESR and SLEDAI score. $\mathrm{ESR}=$ Erythrocyte sedimentation rate, $\mathrm{SLEDAI}=$ Systemic lupus erythematosus disease activity index. ${ }^{* *} \mathrm{p}<0.01$

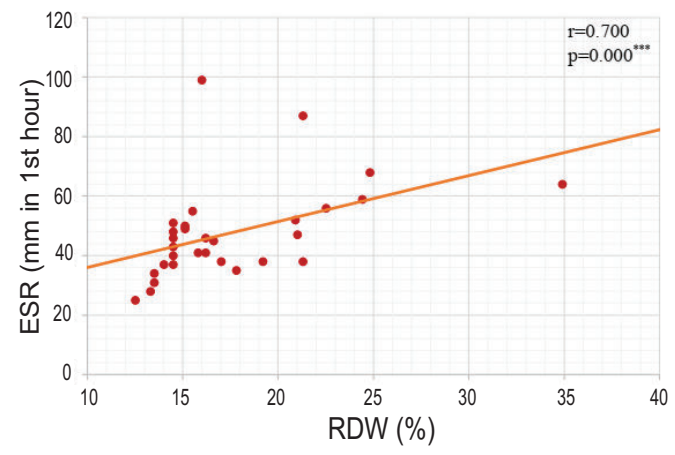

Figure 6: Correlation of RDW with ESR in SLE patients presenting that there is positive correlation between RDW and ESR, RDW $=$ Red cell distribution width, ESR = Erythrocyte sedimentation rate, $\mathrm{SLE}=$ Systemic Lupus erythrematosus, ${ }^{* * *} \mathrm{p}<0.001$ 
Table III : Diagnostic accuracy of RDW and ESR in SLE patients $(\mathrm{n}=30)$

\begin{tabular}{lccccc}
\hline Variables & Sensitivity & Specificity & PPV & NPV & p value \\
\hline RDW & $84.2 \%$ & $72.7 \%$ & $84.2 \%$ & $72.7 \%$ & $0.004^{* *}$ \\
ESR & $78.9 \%$ & $81.8 \%$ & $88.2 \%$ & $69.2 \%$ & $0.001^{* *}$ \\
\hline
\end{tabular}

Statistical analysis was performed and $\mathrm{p}<0.05$ was accepted as level of significance. ${ }^{* *} \mathrm{p}<0.01$. PPV-positive predictive value, NPV - negative predictive value. RDW $=$ Red cell distribution width, ESR = Erythrocyte sedimentation rate, SLE $=$ Systemic Lupus erythrematosus.

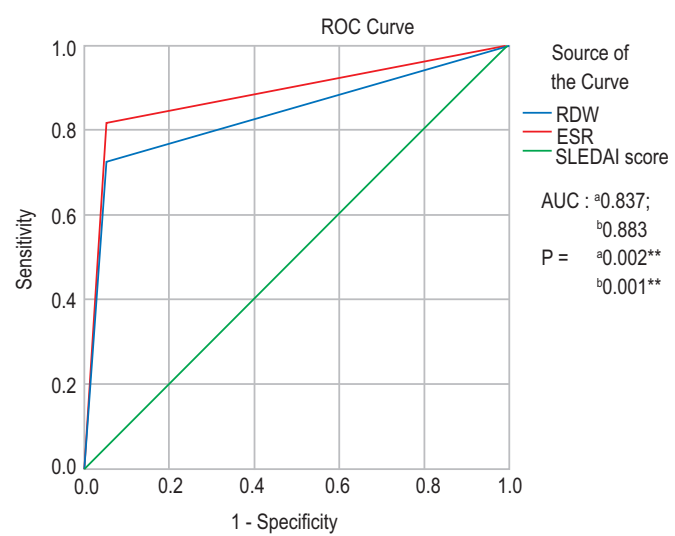

Figure 7: ROC curve showing RDW and ESR in SLE patients $(\mathrm{n}=30)$. ${ }^{\mathrm{a}} \mathrm{RDW}=\mathrm{Red}$ cell distribution width, ${ }^{\mathrm{b}} \mathrm{ESR}=$ Erythrocyte sedimentation rate, $\mathrm{SLE}=$ Systemic Lupus erythematosus. Area Under the Curve (AUC)

\section{Discussion}

Systemic lupus erythematosus (SLE) is an autoimmune disease. It can affect virtually all organs including hematological system. ${ }^{3,20}$ Majority of the patients present with hematological abnormality as their initial and only manifestation. ${ }^{21}$ Some researcher ${ }^{20-22}$ reported an association among hematological abnormalities with active disease. Only a few biomarkers are available for assessing disease activity in SLE. RDW is an ideal biomarker which are easily measured and sensitive to changes in disease activity. ${ }^{15}$ Assessment of disease activity in SLE is vital for evaluating outcomes, differences among SLE patient groups and observing responses to a new drug proposed. ${ }^{23}$ $\mathrm{RDW}$ is a measure of $\mathrm{RBC}$ volume variations. ${ }^{24}$
The present study was undertaken to evaluate RDW in SLE patients as an activity marker.

In present study, RDW and ESR were significantly higher in SLE patients in comparison to those of control. These findings are parallel to the observation of some groups of authors. ${ }^{12,25-26}$

In Multivariate logistic regression analysis showed a significant association among RDW, ESR and active disease (SLEDAI score $>9$ ). Like other inflammatory marker (i.e., ESR) increased RDW was 5 times more risk of developing active disease than inactive SLE. Some researchers of different countries found similar association but different methodology was used in those studies. ${ }^{14,25,27}$

In correlation analysis, RDW and ESR showed positive correlation with active disease and RDW also showed positive correlation with inflammatory marker like ESR in patients with SLE. These findings are corresponding to the observation of some other researchers. ${ }^{11,28}$. It has been demonstrated that RDW is a promising index to estimate the disease activity of SLE. ${ }^{29}$ RDW is an easy and cheap vital inflammatory parameter, the long-life span of red blood cell and RDW may not be affected by recent infections. RDW particularly useful to evaluate the disease activity of SLE patients with infections. ${ }^{28}$

Diagnostic accuracy of RDW and ESR showed almost similar sensitivity and specificity and the area of RDW and ESR under the ROC curve also considered as excellent for separating active SLE

Volume 16 No. 2 December 2021: 95-103 
from inactive SLE patients. No study was found to compare our findings. These might have occurred due to different methodology used in those few studies. However, RDW is recommended as an excellent sensitive inflammatory marker in sever sepsis. ${ }^{29}$

Though the explanation of these changes in RDW and ESR of SLE patients is not known but literature review suggests that increased ESR may be due to chronic inflammatory response with polyclonal increase in immunoglobulins..$^{30-31}$

In SLE, pro-inflammatory cytokines are released during activation of immune cells. These cytokines (interferon) bind with its receptors in the bone marrow, inhibit translation of mRNA, thus inhibit proteins synthesis in hematopoietic cells and inhibit the erythroid progenitor cell proliferation and proerythroblast maturation in the bone marrow. Thus, immature erythrocytes are released into the circulation. ${ }^{32-35}$ On the other hand, proinflammatory cytokines such as interleukin-1 (IL-1), IL-6 and tumor necrosis factor-alpha (TNF- $\alpha$ ) inhibit synthesis or activity of erythropoietin (EPO) and desensitized erythroid progenitors to EPO in bone marrow. Thereby promoting anisocytosis. ${ }^{16,36}$

Some studies reported that genetic and environmental factors may contribute to abnormal activation of immune cells, $\mathrm{T}$ and $\mathrm{B}$ lymphocytes and ineffective regulatory CD4+ and CD8+ T cells. Therefore, sustained autoantibodies are formed in SLE. These auto antibodies cause activation of natural killer (NK) cells. NK cells cause lysis of the target cells. ${ }^{22}$ The hematopoietic system is very much vulnerable to these effects. Thus, autoantibodies cause destruction of circulating erythrocytes leads to a more mixed population of erythrocyte volumes in the circulation. ${ }^{35,37}$ So, inflammation contribute to anisocytosis. Thus, leads to increased RDW. ${ }^{16}$

This increased RDW and ESR might be consequence of increased inflammation process in SLE patients which is evident from its relation to disease activity.

\section{Conclusion}

From results of the study, it can be concluded that increased RDW in systemic lupus erythematosus (SLE) patients can be served as a useful index to estimate the disease activity. RDW testing is inexpensive, widely available and easiest to measure than traditional indicators, such as ESR, CRP and anti-dsDNA levels. However, further prospective and multicenter studies with larger sample size are needed to corroborate the clinical value of RDW in patients with SLE and to determine a cut-off level of RDW for assessing disease activity with a high sensitivity and specificity.

Conflict of interest: None

\section{Acknowledgement}

The authors acknowledge the Department of Medicine Dhaka Medical College, Dhaka for their kind co-operation during sample collections and all the study subjects for their active participation.

\section{References}

1. Mehat P. Pharmaceutical outcomes research to better understand medication non-adherence in patients with systemic lupus erythematosus [Thesis]. Columbia: University of British Columbia 2014; p. 3-4.

2. Bertsias G, Cervera R, Boumpas DT. Systemic lupus erythematosus: pathogenesis and clinical features. Eular textbook on rheumatic diseases. $1^{\text {st }}$ ed. London: BMJ Group 2012; p.476-505.

3. Adelowo OO, Bello MKN. Systemic autoimmune diseases: not so rare in black Africans. J Rheumatol 2014; 4 (1): 1-4.

4. Salehi-Abari I. 2015 ACR/SLICC revised criteria for diagnosis of systemic lupus erythematosus. Autoimmune Dis Ther Approaches 2015; 2 (1): 1-5.

5. Costenbader KH, Feskanich D, Stampfer MJ, Karlson EW. Reproductive and menopausal factors and risk of Systemic Lupus Erythematosus in Women. Arthritis Rheum 2007; 56 (4): 1251-1262. 
6. Sawalha AH, Harley JB, Scofield RH. Autoimmunity and Klinefelter's syndrome: when men have two $\mathrm{X}$ chromosomes. J autoimmune 2009; 33 (1): 31-34.

7. Borchers AT, Keen CL, Shoenfeld Y, Gershwin ME. Surviving the butterfly and the wolf: mortality trends in systemic lupus erythematosus. Autoimmun Rev 2004; 3 (6): 423-453.

8. Jacobsen S, Petersen J, Ullman S, Junker P, Voss A, Rasmussen JM, Tarp U, Poulsen LH, van Overeem Hansen G, Skaarup B, Hansen TM, Pødenphant J, Halberg P. Mortality and causes of death of 513 Danish patients with systemic lupus erythematosus. Scand J Rheumatol 1999; 28 (2): 75-80.

9. Jakes RW, Bae SC, Louthrenoo W, Mok CC, Navarra SV, Kwon N. Systematic review of the epidemiology of systemic lupus erythematosus in the Asia-Pacific region: Prevalence, incidence, clinical features and mortality. Arthritis Care Res 2012; 64 (2): 159-168.

10. Navarra SV, King JO. An overview of clinical manifestations and survival of systemic lupus erythematosus patients in Asia. APLAR J Rheumatol 2006; 9: 336-341.

11. Xie S, Chen X. Red blood cell distribution width-toplatelet ratio as a disease activity-associated factor in systemic lupus erythematosus. Medicine (Baltimore) 2018; 97 (39): 1-7.

12. Mohamed OSD, Azmy GJ, Abu Elfad EM. Clinical significance of red blood cell distribution width in systemic lupus erythematosus patients. Egypt Rheumatol Rehabil 2020; 47 (38): 1-8.

13. Lappegård J. Red cell distribution width (RDW) and risk of arterial cardiovascular disease - A Literature Study [Thesis]. Norway: University of Tromsø. 2016; p. 7-10.

14. Lippi G, Cervellin G, Sanchis-Gomar F. Red blood cell distribution width: A marker of anisocytosis potentially associated with atrial fibrillation. World J Cardiol 2019; 11 (12): 292-304

15. Shoeib SA, Abd Elhafez MA, Abd El Hamed AE, Sherief HG. Red blood cell distribution width to estimate lupus activity. Menoufia Med J 2018; 31 (2): 449-454.

16. Ozisler C, Sandikci SC. Evaluation of red blood cell distribution width in patients with psoriatic arthritis. Egypt. Rheumatol 2018; 42 (2020): 309312 .
17. Gladman DD, Ibañez D, Urowitz MB. Systemic lupus erythematosus disease activity index 2000. J Rheumatol 2002; 29 (2): 288-291.

18. Hill VL, Simpson VZ, Higgins JM, Hu Z, Stevens RA, Metcalf JA, Baseler M. Evaluation of the Performance of the Sysmex XT-2000i Hematology Analyzer with Whole Bloods Stored at Room Temperature. Lab Med 2009; 40 (12): 709-718.

19. Ghai CL. A Textbook of Practical Physiology. 8th ed. India: Jaypee. 2010; p. 93-98.

20. Aleem A, Al-Arfaj, Khalil N, Alarfaj H. Haematological abnormalities in systemic lupus erythematosus. Acta Reumatol Port 2014; 39 (3): 236-241.

21. Sasidharan PK, Bindiya M, Sajeeth-Kumar KG. Systemic lupus erythematosus- a hematological problem. J Blood Disord Transfus 2013; 4 (6): 1-4.

22. Agrawal SR, Tiewsoh I, Rajput A, Jain A. A cross sectional hospital-based study of clinical and immunological profile of systemic lupus erythematosus patients from central rural India. Indian J Allergy Asthma Immunol 2013; 27 (1): 33-37.

23. Romero Diaz J, Isenberg D, Ramsey Goldman R. Measures of adult systemic lupus erythematosus: Updated Version of British Isles Lupus Assessment Group (BILAG 2004), European Consensus Lupus Activity Measurements (ECLAM), Systemic Lupus Activity Measure, Revised (SLAM R), Systemic Lupus Activity Questionnaire for Population Studies (SLAQ), Systemic Lupus Erythematosus Disease Activity Index 2000 (SLEDAI 2K), and Systemic Lupus International Collaborating Clinics/American College of Rheumatology Damage Index (SDI). Arthritis Care Res 2011; 63 (11): 37-46.

24. Bujak K, Wasilewski J, Osadnik T, Jonczyk S, $\mathrm{Ko}^{3}$ odziejska A, Gierlotka M, Gasior M. The prognostic role of red blood cell distribution width in coronary artery disease: a review of the pathophysiology. Dis Markers 2015; 2015: 1-12.

25. Vayá A, Alis R, Hernández JL, Calvo J, Micó L, Romagnoli M, Ricarte JM. RDW in patients with systemic lupus erythematosus. Influence of anaemia and inflammatory markers. Clin Hemorheol Microcirc 2013; 54 (3): 333-339.

26. Gulkesen A, Gozel N. Can number of platelets, blood red cell distribution, volume of neutrophil/ lymphocyte ratio and mean platelet volume in

Volume 16 No. 2 December 2021: 95-103 
patients with systemic lupus erythematosus and sjögren be used as an inflammation marker? MedScience 2019; 8 (1):106-108.

27. Reategui-Sokolova C, Ugarte-Gil M, GamboaCárdenas R, Zevallos F, Cucho Venegas J, AlfaroLozano J, Medina M, Rodríguez-Bellido Z, PastorAsurza C, Alarcün G, Perich-Campos R. RDW levels are associated with damage accrual in systemic lupus erythematosus patients. BMJ 2017; 76 (2): 1-2.

28. Hu ZD. Red blood cell distribution width: a promising index for estimating activity of autoimmune disease. J Lab Precis Med 2016; 1 (2): $1-4$.

29. Jandial A, Kumar S, Bhalla A, Sharma N, Varma N, Varma S. Elevated red cell distribution width as a prognostic marker in severe sepsis: A prospective observational study. Indian J Crit Care Med 2017; 21 (9): 552562.

30. Hamza KM, Bashir ZM. Hematological profile of Sudanese patients with systemic lupus erythematosus. Clin Med J 2016; 2 (4): 40-45.

31. Al-Arfaj AS, Khalil N. Clinical and immunological manifestations in 624 SLE patients in Saudi Arabia Lupus 2009; 18 (5): 465-473.
32. Levinson $\mathrm{W}$, editor. Review of medical microbiology and immunology. $9^{\text {th }}$ ed. New York: McGraw-Hill companies 2004; p. 401-424.

33. Liu H, Ozaki K, Matsuzaki Y, Abe M, Kosaka M, Saito S. Suppression of haematopoiesis by $\operatorname{IgG}$ autoantibodies from patients with systemic lupus erythematosus (SLE). Clin Exp Immunol1995; 100 (3): $480-485$.

34. Fayyaz A, Igoe A, Kurien BT, Danda D, James JA, Stafford HA, Scofield RH. Hematological manifestations of lupus. Lupus sci Med 2015; 2 (1): $1-18$.

35. Giannouli S, Voulgarelis M, Ziakas PD, Tzioufas AG. Anemia in systemic lupus erythematosus: from pathophysiology to clinical assessment. Ann Rheum Di 2006; 65 (2): 144-148.

36. He Y, Liu C, Zeng Z, Ye W, Lin J, Ou Q. Red blood cell distribution width: a potential laboratory parameter for monitoring inflammation in rheumatoid arthritis. Clin Rheumatol 2018; 37 (1):161-167.

37. Kumar V, Abbas AK, Fausto N, editors. Robins and Cotran pathologic basic of disease. $7^{\text {th }}$ ed. China: Elsevier Saunders 2015; p. 194-230. 